\title{
In Situ Study of Mechanical Testing and Fracture Process of Glassy Polystyrene Grafted Nanoparticle Assembly: Impact of Film Thickness and Strain Rate
}

\author{
Ming-Siao Hsiao ${ }^{1,2}$, Yang Jiao ${ }^{1,2}$, Robert Wheeler ${ }^{1,2}$, Joseph Lefebvre ${ }^{3}$, Sanjit Bhowmick ${ }^{3}$, Richard A. \\ Vaia $^{1}$ and Lawrence F. Drummy ${ }^{1}$ \\ ${ }^{1 .}$ Materials and Manufacturing Directorate, Air Force Research Laboratories, WPAFB, OH \\ 2. UES, Inc., Dayton, OH \\ 3. Bruker Corporation, Minneapolis, $\mathrm{MN}$
}

Three dimensional assemblies of polymer-grafted "hairy" nanoparticles (HNPs) are of current interest for a wide array of mechanical and electrical applications. The areal grafting density of the polymer chains on the nanoparticle surfaces, and the molecular weight of the grafted polymers, determine the resulting interparticle spacing and volume fraction of the polymer and nanoparticle constituents. Because the polymer is chemically grafted to the particle surface particle-particle contacts are eliminated, as opposed to a physical blending process in which nanoparticle agglomeration is typically observed. The mechanical properties of HNPs films are therefore dominated by the polymer-polymer entanglements and the ordering in the nanoparticle assembly. Previous studies of HNP thin films have largely relied on nanoindentation, due to the challenges of preparing sub-micron testing specimens [1]. The emergence of microelectromechanical systems (MEMS) techniques for fabricating devices and microscale specimen preparation using focus ion beam (FIB) allows for in situ study of failure process of HNP assemblies. Previous results in ex-situ tests [2] have shown that crack processes dominate deformation in HNP assemblies with low grafting density, but crazing occurs for assemblies with high grafting density. As with linear polystyrene, molecular weight and strain rate also impact the transition from crack to craze. These correlations between HNP architecture and subsequent failure mode refines the HNP design space for the synthesis and fabrication of assemblies with defined mechanical properties. In this study fundamental processes of craze initiation, the effect of film thickness, and the role of silica nanoparticles are examined in polystyrene-silica HNP assemblies synthesized using the grafting-from method.

In situ mechanical tests of HNP films examined the role of film thickness (200 nm to $1300 \mathrm{~nm}$ ) respectively at fixed strain rate by simultaneous collection real time load-displacement data using SEM imaging. Table 1 shows the molecular characterization and thermal analysis of grafted polystyrene $\left(\mathrm{T}_{\mathrm{g}}{ }^{\mathrm{PS}}\right)$ for this HNP assembly. Fig. 2 shows HAADF-STEM images of aHNP-PS-1 with a nominal film thickness of 150 and $1300 \mathrm{~nm}$, indicating particle-particle agglomeration is not present in either case. Fig. 3 shows SEM images and strain-stress profiles of two PS grafted HNPs testing specimens with thicknesses of 150 $\mathrm{nm}(\mathrm{a}-\mathrm{c})$ and $1300 \mathrm{~nm}(\mathrm{~d}-\mathrm{f})$ prepared by FIB milling. The tests were run at displacement-controlled mode with a strain rate of $\sim 4 \times 10^{-3} \mathrm{~s}^{-1}$. Yield stress and elastic modulus were measured to be 73 to $106 \mathrm{MPa}$ and 634 to $930 \mathrm{MPa}$ for thin HNP film and $48 \mathrm{MPa}$ and $585 \mathrm{MPa}$ for thick film case, compared to $22 \mathrm{MPa}$ and $3000 \mathrm{MPa}$ for PS thin film $(100 \mathrm{~nm}$ ) (nanoindentation). SEM videos showed cracks dominated in both micrometer size film cases at this high strain rate. Our study showed in situ study of mechanical test of these defect free micrometer scale HNP films is feasible through minimizing FIB milling and electron beam effects on the specimens.

\section{References:}

[1] Ramachandramoorthy, R., Bernal, R., and Espinosa, H. D. ACS Nano 9 (2015), p. 4675. 
[2] Hsiao, M. S. et al, Microsc. Microanal. 22(S3) (2016), p. 1856.

Table 1. Molecular and thermal characterizations of the PS grafted hairy silica nanoparticles assemblies

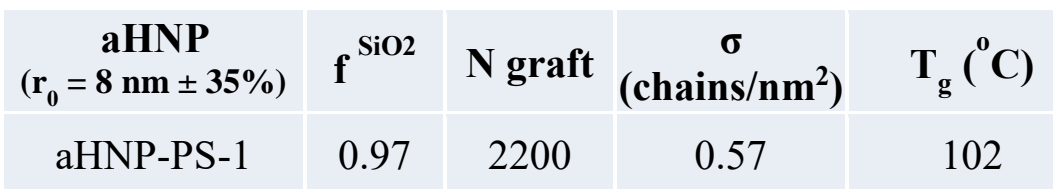
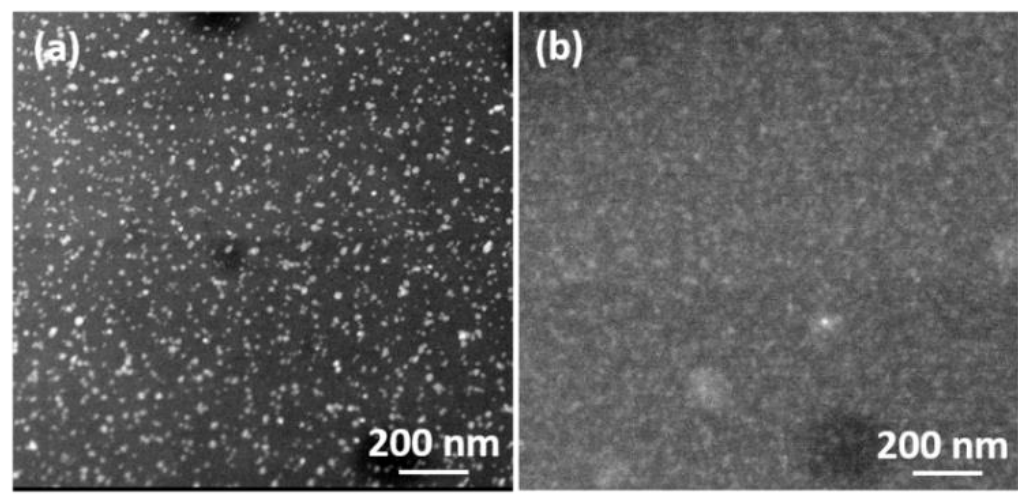

Figure 2. HAADF-STEM images of (a) aHNP-PS-1films with $150 \mathrm{~nm}$ thickness and (b) $1300 \mathrm{~nm}$ thickness, respectively. White dots are silica nanoparticles.
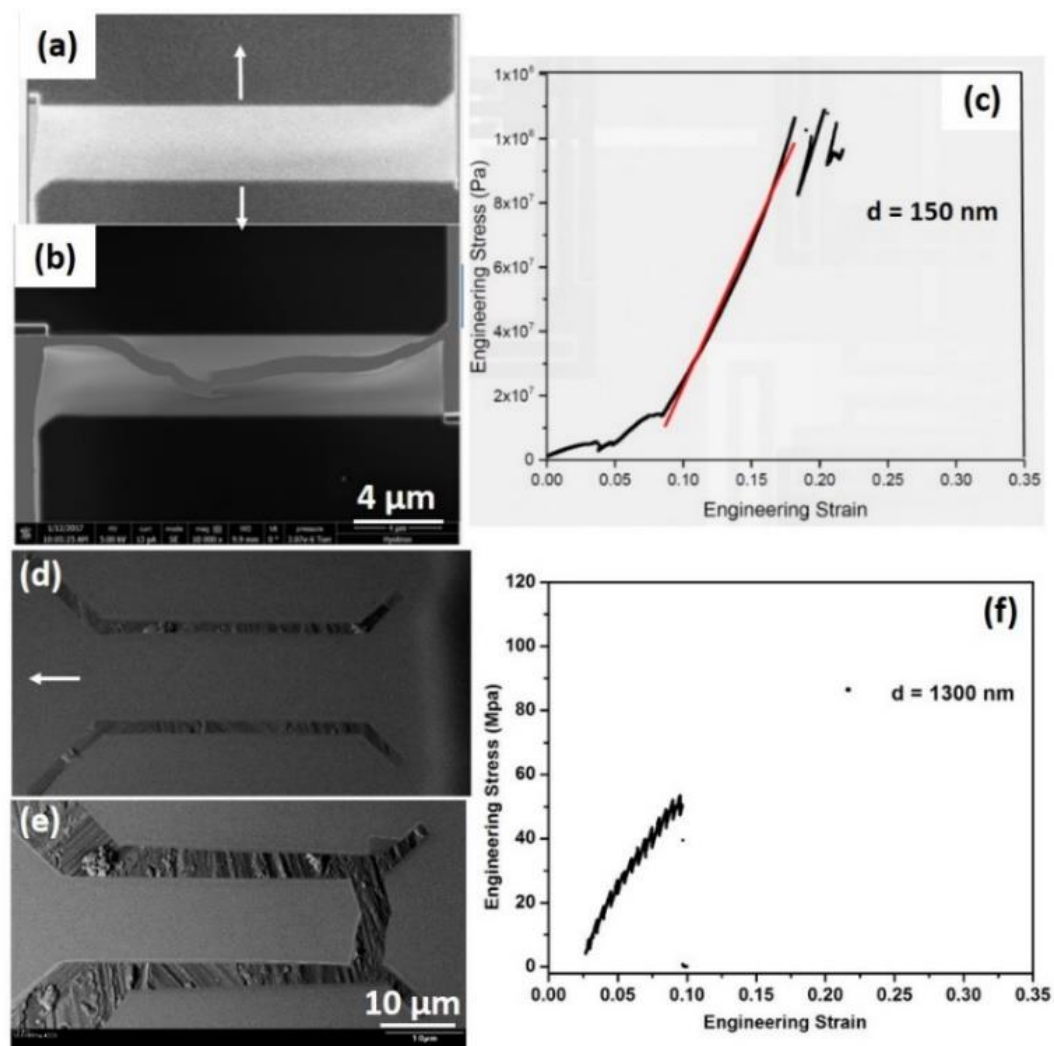

Figure 3. SEM images and strain-stress relationship for micrometer size aHNP-PS-1 thin film $(d=150$ $\mathrm{nm})(\mathrm{a}-\mathrm{c})$ and thick film $(\mathrm{d}=1300 \mathrm{~nm})(\mathrm{d}-\mathrm{f})$ with a strain rate of $\sim 4 \times 10^{-3} \mathrm{~s}^{-1}$. Crack formed in both specimens $(b, e)$. White arrow head indicates strain direction of test specimen. 